\title{
Self-healing Control Algorithms for Distribution Networks
}

\author{
Huan-Yuan Wei ${ }^{1, a}$, Jin-Bo Lan², Yan-Fei Ye ${ }^{1}$, Xiao-Hui Li ${ }^{1}$, Jian-Fei Yang ${ }^{1}$ \\ ${ }^{1}$ School of Electrical and Automation Engineering, Nanjing Normal University, China \\ ${ }^{2}$ Guodian Nanjing Automation co.,LTD, Nanjing 211100, China \\ ae-mail:js_weihuanyuan@163.com
}

Keywords: Self-healing, adjacency matrix, distribution network.

\begin{abstract}
Self-healing is an important feature of intelligent distribution networks. Self-healing includes the abilities of self-perception, self-diagnosis, self-decision and self-recovery. In this paper, a adjacency matrix of the distribution network is used to determine the network fault perception and make a decision. The power supply is then restored utilizing network reconfiguration. The isolated nodes in the network are identified using the node matrix of the distribution network. A new network is found according to the contact characteristics between nodes so as to restore power. Finally, the feasibility of the algorithm is verified via MATLAB simulations.
\end{abstract}

\section{Introduction}

In recent years, there have been instances of large power failures both in China and abroad. These accidents have caused people to pay attention to the reliability of the distribution networks. The concept of self-healing of distribution networks has come into focus. "Self-healing" is source in the biomedical field, which is defined for system of a self-aware state, without manual intervention in self-judgment and taking appropriate measures to restore to the normal state. Grid self-healing means that there is no need, or only a small need, for human intervention. Instead, advanced monitoring methods for power-grid operation states are utilized in order to make continuous on-line diagnosis and assessment. This enables timely detection and quick adjustment of problems, eliminating hidden problems. Grid self-healing includes the abilities of self-perception, self-diagnosis, selfdecision and self-recovery, and makes safe and reliable operation of distribution networks in different kinds of conditions possible. The process of self-healing of the smart grid can adjust the different states of the network. It leads to optimization and warning under normal conditions and fault diagnosis under faulty conditions; thereby making reconfiguration of the network and restoration of the power supply possible. Self-healing even makes separation from the main grid under extreme conditions possible. Under this condition, continued operation with a distributed power and energy storage device is achieved.

The key technologies pertaining to a distribution network's self-healing and the structure of the whole self-healing system are introduced in the paper [1]. The scheme of self-healing control for power networks includes the centralized control mode, the decentralized control mode and the centralized/decentralized coordinated control method [2]. This paper discusses the state estimation of a power network during the process of network self-healing, but there is no further study on how to do the self-healing of the power network in the paper [3]. A self-healing control framework of "2-36" is proposed in the paper [7], namely, the two loop control logic, the three layer control structure, and the six control links. The design focuses on the contradiction between the fast and the global response of the local dynamic process of the network. A local control with quick reaction and a global control with relatively slow reaction are made and the coordination layer is joined between the two loops control logic in order to solve the contradiction between the global control and local control. In this paper, the station of the system relying mainly on with advanced analysis and calculation function of master will collect the amount of measurement information, through the analysis of the calculation to determine fault types, fault location and formation control decision, issued to protection device. We focus on a method for centralized control through the adjacency matrix of the distribution network used to find the network reconfiguration path after a failure. Analyze the exist- 
ence of isolated nodes in the network, which are used as the starting of the fault self-healing strategy.

\section{Distribution Network Node Matrix}

A distribution network is characterized by closed-loop design and open-loop operation. A large number of segmented switches which are often closed in order to isolate faults, as well as a small number of contact switches which are often open in order to provide optional power supply, are combined in the network. Therefore, under normal operating conditions, the switching states can be changed according to different load conditions in order to adjust the network structure. In order to achieve control, information on the structure and the current operating state of the distribution network is needed. This can provide the information for the next self-healing program, and make diagnosis and control possible. We therefore need to define two matrices. One depicts information on the current state, which can evaluate whether the distribution network has a failure or not. The other matrix presents the structure of the distribution network; meaning, whether or not the nodes are connected. This is done in order to choose a feasible and available self-healing scheme for the distribution network. An example of a distribution network is shown in Figure 1.

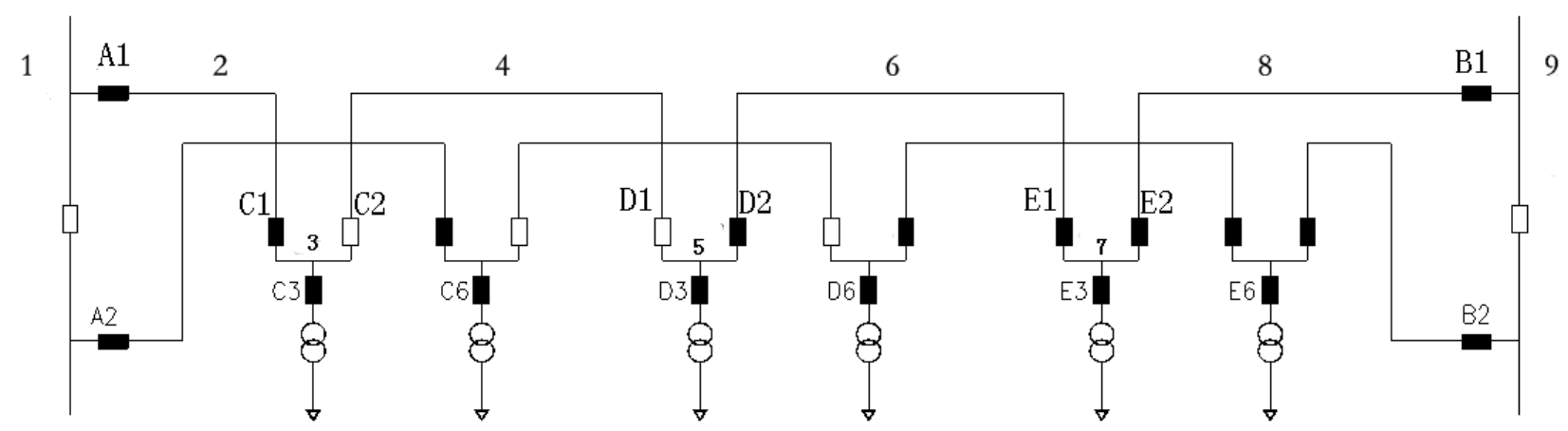

Fig.1 Hand-in-hand distribution network

In the distribution network, the establishment of the power distribution network connectivity is set which the lines are equivalent to the nodes and the circuit breakers are equivalent to the edges. The connected graph is undirected graph and the weights are set to 1 .

According to the structural diagram of the distribution network, the matrix of the current distribution network structure is generated according to generation of nodes. Set $A=\left\{a_{1}, a_{2} \ldots, a_{n}\right\}$, and define matrix Con $=\left(\operatorname{Con}_{i 1}\right)_{n \times n}$ :

$$
\operatorname{Con}_{t i}=\left\{\begin{array}{l}
0_{i} a_{t} \text { and } a_{f} \text { are not comrected } \\
1, a_{i} \text { and } a_{f} \text { are comnected }
\end{array}\right.
$$

The matrix generated by Figure 1 then becomes:

$$
\text { Con }=\left[\begin{array}{lllllllll}
1 & 1 & & & & & & & \\
1 & 1 & 1 & & & & & & \\
& 1 & 1 & 0 & & & & & \\
& & 0 & 0 & 0 & & & & \\
& & & 0 & 1 & 1 & & & \\
& & & & 1 & 1 & 1 & & \\
& & & & & 1 & 1 & 1 & \\
& & & & & & 1 & 1 & 1 \\
& & & & & & & 1 & 1
\end{array}\right]
$$

Then the distribution network circuit breaker and switch all closed and a path matrix is generated according to the distribution. The rule is similar to the matrix Con. So it can provide a path for the power distribution network restoration. The matrix generated by Figure 1 then becomes: 


$$
\text { State }=\left[\begin{array}{lllllllll}
0 & 1 & & & & & & & \\
1 & 0 & 1 & & & & & & \\
& 1 & 0 & 1 & & & & & \\
& & 1 & 0 & 1 & & & & \\
& & & 1 & 0 & 1 & & & \\
& & & & 1 & 0 & 1 & & \\
& & & & & 1 & 0 & 1 & \\
& & & & & & 1 & 0 & 1 \\
& & & & & & & 1 & 0
\end{array}\right]
$$

\section{Self-healing Program Flow}

The self-healing ability of a distribution network needs to include continuous monitoring of its operational processes. Through the continuous monitoring of the node matrix, the power failure of the power network is diagnosed. When the power failure occurs in the distribution network, the new network structure is adjusted in accordance with the original distribution network structure, thus restoring the power supply. Developing many kinds of reconfiguration schemes will facilitate finding the best scheme according to the constraints of the system. During this process, the short-circuit in the power supply network can be found and excluded by utilizing the appropriate circuit breaker in the network. When the state of the circuit breaker is inconsistent with the normal operational state, it can be considered as a short-circuit circuit and be excluded. This specific process is shown in Figure 2.

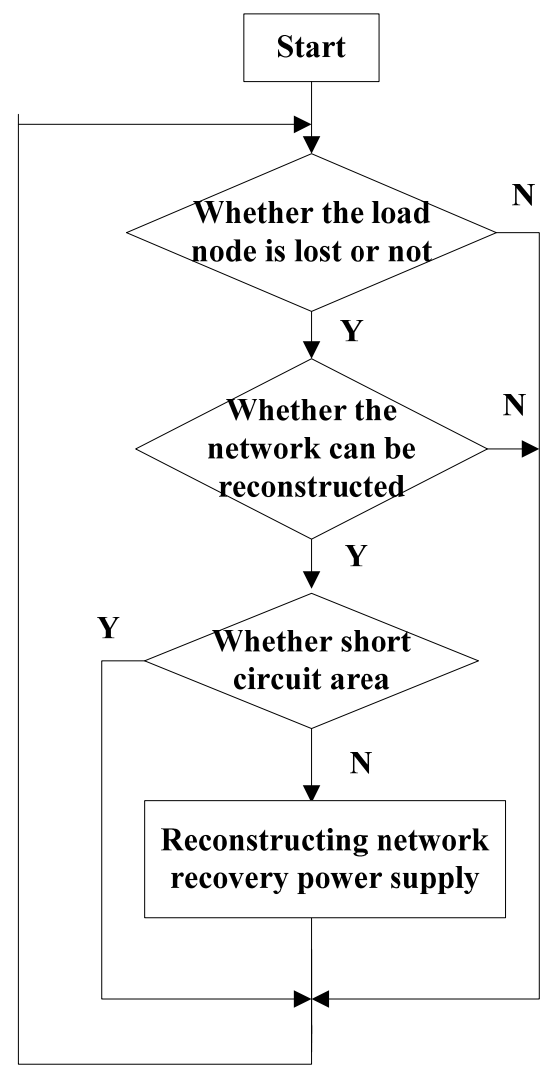

Fig.2 Self-healing flow chart

Assuming that Node 2 has a short-circuit, then Node 3 loses the voltage. Algorithm searches for connected and charged nodes from both sides of Node 3 follow the path presented in Figure 3. 


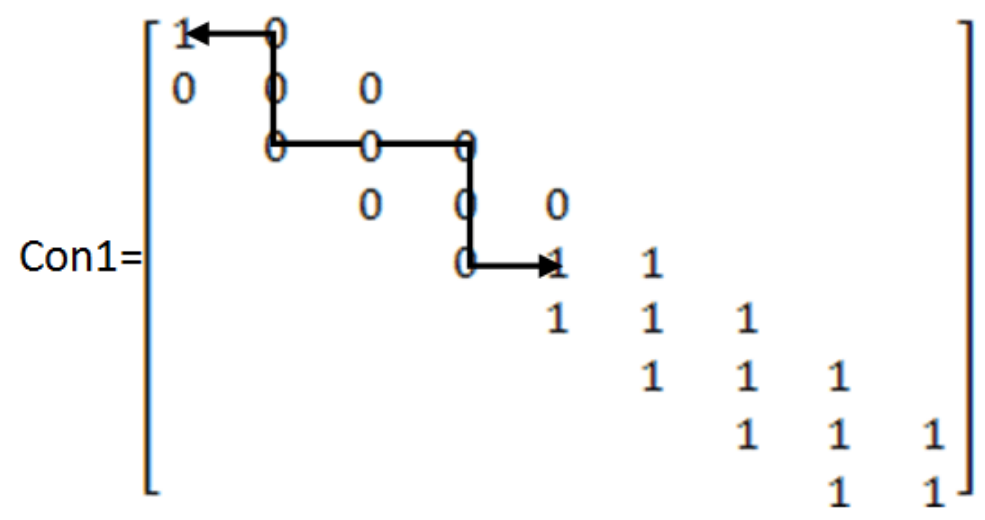

Fig.3 Searching for paths

The algorithm search following the path dictated by the state of the matrix shows two loops. One is connected with Node 1 and the other is connected with Node 5. Then, in comparison with the Con matrix: If the connection between the nodes is changed, the contact switch state is changed. For instance, Node 4 and Node 5, and Node 2 and Node 3 are not the same as before. Therefore, Node 3 can only connect with Node 4 and Node 5.

In this paper, the distribution network model was built using MATLAB. The state of the circuit breaker is simulated by the S-function, and the state of the circuit breaker is transmitted to the control function. Then a power supply network is found by controlling the function. The feasibility of the scheme is verified by the S-function, and the model is shown in Figure 4.

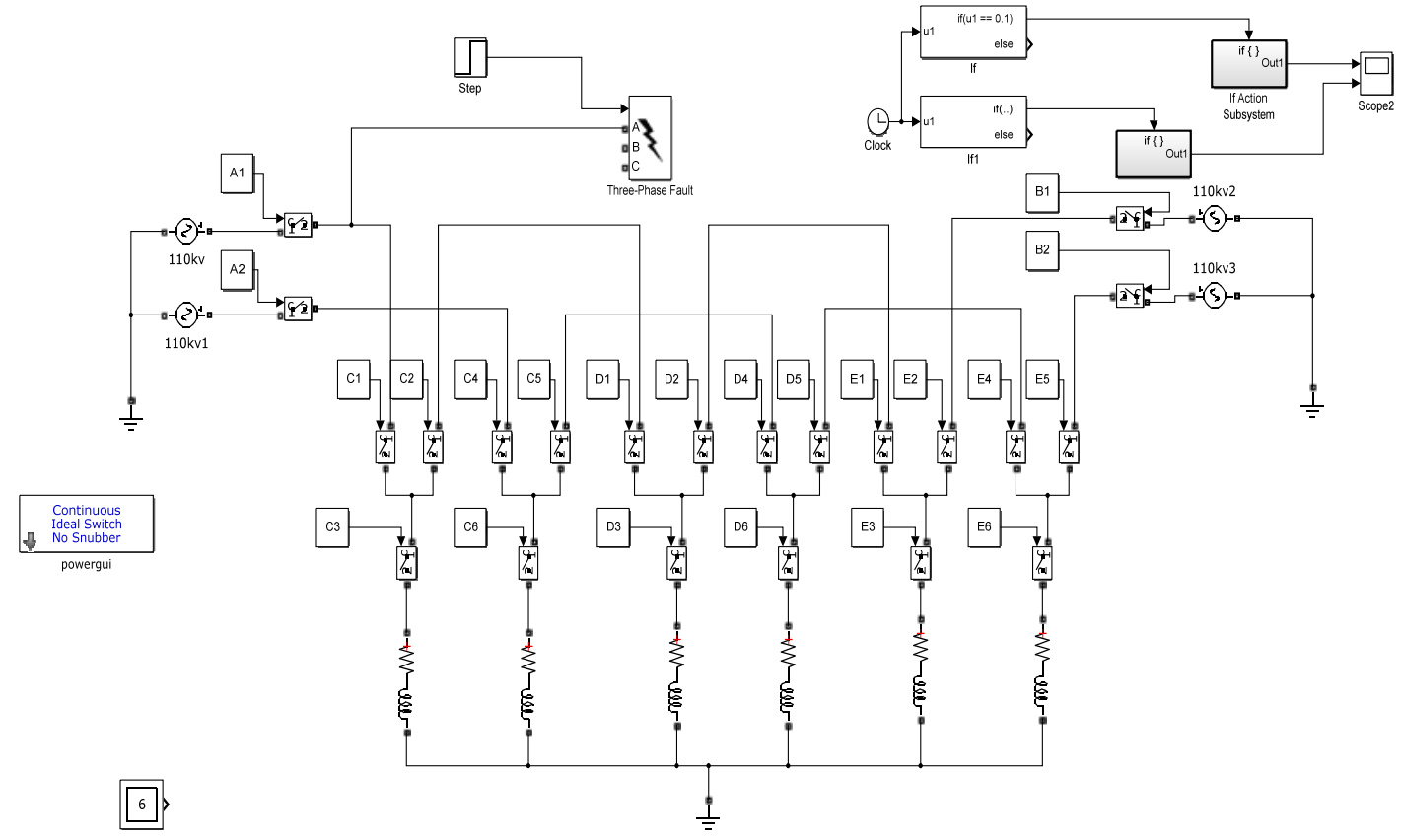

Fig.4 MATLAB simulation chart

Through the construction of the distribution network in Figure 1, a short circuit is simulated. It is verified by simulation that the network can recover the power supply. Through simulation of 4 normal operation modes, the circuit breaker action in Table 1 is obtained. We can find that the algorithm can reconstruct the network and restore the power supply through the closing circuit breaker.

Table 1 Simulation results

\begin{tabular}{|r|c|c|}
\hline & $\begin{array}{c}\text { The closed circuit breaker } \\
\text { under normal operation }\end{array}$ & $\begin{array}{c}\text { The closed circuit breaker for simulation of } \\
\text { short circuit }\end{array}$ \\
\hline 1 & A1,C1,D2,E1,E2,B1 & A1,C1,C2,D1,E2,B1（Node 6 short circuit) \\
\hline 2 & A1,C1,D2,E1,E2,B1 & C2,D1,D2,E1,E2,B1（Node 2 short circuit) \\
\hline 3 & A1,C1,C2,D1,E2,B1 & A1,C1,D2,E1,E2,B1（Node 4 short circuit) \\
\hline 4 & A1,C1,C2,D1,E2,B1 & A1,C1,C2,D1,D2,E1（Node 8 short circuit) \\
\hline
\end{tabular}




\section{Summary}

In this paper, a self-healing scheme is developed by utilizing a node matrix in order to complete the distribution network's self-decision and self-diagnosis. The path of the distribution network selfhealing can be found and the current state of the distribution network can be known through the adjacency matrix. Searching process is simple and do not need to search every line. Because the distribution network is simple, no other constraints are needed. When the distribution network structure is complex, it is recommended to choose and optimize according to the power network voltage constraints and power loss constraints from the multiple networks of recovery power supply that are found. Thereby, a more reliable recovery power supply can be established. Under the background of the construction of the intelligent distribution network in China, the research is gradually deepened. It is the key problem in the future to find out the best solution in the process of power network selfhealing.

\section{Acknowledgement}

This work was financially supported by the National-Natural Science Foundation of China (51177074 \& 51407095).

\section{References}

[1] Song Han, Biming Tian, Elizabeth Chang: Efficient Threshold Self-Healing Key Distribution with Sponsorization for Infrastructureless Wireless Networks. IEEE Transactions on Wireless Communications, 8(4): 1876-1887 (2009)

[2] Qin Hongxia, Tan Zhihai: Research and design of self-healing control system for smart distribution network. Power System Protection and Control, 42(22): 134-139 (2014)

[3] Haibo You, Vijay Vittal, Zhong Yang: Self-Healing in Power Systems: An Approach Using Islanding and Rate of Frequency Decline-Based Load Shedding. IEEE Transactions on Power Systems, 18(1): 174-181 (2003)

[4] Dong Xuzhu, Huang Shaoyuan, Chen Rouyi: Self-healing control technology for smart distribution network. Power System Automation, 36(18): 17-21 (2012)

[5] Ren Jiangbo, Guo Zhizhong: Study on the state estimation mode of the power network selfhealing control. Power Network Technology, 31(3): 59-62 (2007)

[6] Alexandre Mendes, Natashia Boland, Patrick Guiney, Carlos Riveros: Switch and Tap-Changer Reconfiguration of Distribution Networks Using Evolutionary Algorithms. IEEE Transactions on Power Systems, 28(1): 85-92 (2013)

[7] Guo Zhiyong: Power network self-healing control scheme. Power System Automation, 29(10): 85-91 (2005)

[8] Sanjay Bahadoorsingh, Jovica V. Milanovic', Yan Zhang, et al: Minimization of Voltage Sag Costs by Optimal Reconfiguration of Distribution Network Using Genetic Algorithms. IEEE Transactions on Power Delivery, 22(4): 2271-2278 (2007)

[9] Daniel Pinheiro Bernardon, Vinicius Jacques Garcia, Adriana Scheffer Quintela Ferreira, Luciane Neves Canha: Multicriteria Distribution Network Reconfiguration Considering Subtransmission Analysis. IEEE Transactions on Power Delivery, 25(4): 2684-2691 (2010)

[10] Chen Xingying, Gu Xinxin, Yu Kun: Self-healing control system structure of urban power network. Power System Automation, 33(24): 38-42 (2009)

[11] Ao Zhigang: Artificial intelligence and expert system. Beijing: Press of machinery industry (2010) 
[12] Zhou Men: Study on hybrid control of self-healing based on Control Theory. North China Electric Power University (2013)

[13] Liu Xi: Research on the self-healing strategy of distribution network based on immune algorithm and fuzzy evaluation. North China Electric Power University (2012)

[14] Liu Bosi, Xie Kaigui, Zhou Jiaqi: Dynamic programming algorithm for distribution network reconfiguration. Chinese Journal of Electrical Engineering Engineering, 25(9): 29-34 (2005)

[15] Hu Wen, Sun Yunlian, Zhang Wei: Research on the reconfiguration of smart distribution network based on the improved adaptive genetic algorithm. Power system protection and control, 41(23): 85-90 (2013)

[16] Amin A. EPRI/DOD complex interactive network/systems initiative:self-healing infrastructure[C]. Proceedings of 2nd DA RPA-JFACCSymp Advances in Enterprise Control Los Alamitos IEEE ComputerSoc Press,USA (2000)

[17] You H, Vittal V, Yang Z. Self-healing in Power System: an Approachusing Islanding and Rate of Frequency Decline-based Load Shedding[J]. IEEE Transactions on Power Systems, 18(1):174-181 (2003)

[18] Chicco G,Napoli R,Postolache P,et al.Customer Characterizationoptions for Improving the Tariff Offer[J]. IEEE Transactions on Power Systems,18(1):381-387 (2003) 